mehr für gleichgültig halten können, wieviel Brunnen getrunken wird und was daneben noch an Salzen durch Nahrung und Getränk aufgenommen wird. Hier beginnt des Praktikers, des Badearztes Domäne: die Wichtigkeit und Notwendigkeit ärztlicher Beaufsichtigung aller Badenden, nicht zum wenigsten auch die Einhaltung bestimmter Diätformen ist, das zeigen die obigen Ausführungen, gerade durch die neuere wissenschaftliche Entwicklung der Pharmakologie erwiesen worden. Freuen wir uns, daB auch in diesem Falle wieder die ärztliche Praxis der wissenschaftlichen Theorie vorausgeeilt ist. Freuen wir uns besonders auch darüber, daß die Heilquellen, die mit so üppiger Mannigfaltigkeit dem Füllhorn der Natur entfließen, die sachverständigen Ärzte gefunden haben, die als treue Hüter dieser Bodenschätze, sie zum Segen der Menschheit zu verwenden wissen.

Das Verhältnis zwischen Balneologie und Pharmakologie ist letzten Endes nur ein typischer Spezialfall eines in der Entwicklung der Wissenschaften häufig auftretenden, allgemeinen Phänomens: und so können die Beziehungen beider Disziplinen nicht eindrucksvoller dargestellt und als Spezialfall gekennzeichnet werden als mit Hilfe einiger Sätze, die der leider viel $\mathrm{zu}$ früh verstorbene Nationalökomom MAX WEBER in ganz anderem Zusammenhang geprägt hat. In seinem Aufsatz über die Objektivität sozialwissenschaftlicher und sozialpolitischer Erkenntnis schreibt er: „Nicht die sachlichen Zusammenhänge der Dinge, sondern die gedanklichen Zusammenhänge der Probleme liegen den Arbeitsgebieten der Wissenschaft zugrunde: wo mit neuer Methode einem neuen Problem nachgegangen wird und dadurch Wahrheiten entdeckt werden, welche neue, bedeutsame Gesichtspunkte eröffnen, da entsteht eine neue ,Wissenschaft:" (Gesammelte Aufsätze zur Wissenschaftslehre, Seite I66.)

Auch Pharmakologie und Balneologie hatten, trotz ihrer sachlichen Zusammenhänge, jede ihre eigenen gedanklichen Probleme und ihre ganz verschiedenen Methoden. Das Problem Mineralwässer und die Art ihrer Wirkung lag den Gredankengängen und der analytischen Arbeitsweise der experimentellen Pharmakologie zunächst fern. Erst nachdem mit neuen Gesichtspunkten und Methoden für das Problem der Salzwirkungen und Ionenverschiebungen neue gedankliche Zusammenhänge hervortraten, ist hier, das sollen die obigen Ausführungen zeigen, ein neues Kapitel biologischer Wissenschaft, einer ,,denkenden Ordnung der empirischen Wirklichkeit" entstanden, wo sich, was vorher getrennt war, harmonisch zu gegenseitiger Förderung vereinigen kann und vereinigen wird.

\title{
ORIGINALIEN.
}

\section{CHIRURGISCHE EINGRIFFE BEI AKUTER UND SUBAKUTER LEBERATROPHIE*).}

Von

Prof. Dr. W. Braun.

Aus der I. Chirurgischen Abteilung des Stadt. Krankenhauses in Friedrichshain-Berlin.

Die schweren degenerativen bzw. nekrotisierenden Parenchymerkrankungen der Leber, welche man gemeinhin, nicht immer allerdings zutreffend, nach der hervorstechendsten pathologisch-anatomischen Folge, dem Leberschwund, als aleute oder subakute Leberatrophie ${ }^{1}$ ) bezeichnet, gehörten in der Vorkriegszeit bei uns zu den größten Seltenheiten. In der Nachkriegszeit haben sie, ebenso wie die Leber- und Gallensystemerkrankungen überhaupt, in beunruhigendem Maße zugenommen. Auch wir Chirurgen müssen uns auf diese Leberparenchymerkrankungen einstellen. Wir müssen wissen, was wir in solchen Fällen zu tun oder zu lassen haben.

Die schwersten Formen der akuten Leberatrophie, welche bei raschem Leberschwund unter dem Bilde der hepatischen Vergiftung und der Leberinsuffizienz fast stets in wenigen Wochen im cholämischen Coma zugrunde gehen, berühren den Chirurgen weniger wie den pathologischen Anatomen und den Internisten. Anders steht es mit den gleichartigen, weniger stürmisch verlaufenden Parenchymerkrankungen. Hier gibt es Fälle verschiedener Atiologie, in welchen bei starkem Ikterus, fehlender oder geringer Leberverkleinerung eine Gallensteinanamnese, Koliken, Fieber bis zu Schüttelfrösten, heftige Leberschmerzen oder Leberdruckschmerz beobachtet werden. Auch mit den besten diagnostischen Hilfsmitteln kann es hier unmöglich sein, die Differentialdiagnose zwischen Choledochussteinverschluß oder aufsteigender Cholangitis einerseits und primärer Parenchymerkrankung der Leber anderseits zu stellen. Eine Cholangitis ist nicht selten der Vorläufer oder das bedingende Moment einer Leberatrophie; ein Steinleiden oder eine Cholangitis kann sich mit der Parenchymnekrose der Leber verbinden. Wie schwer die Entscheidung sein kann; erhellt daraus, daß z. B, einer der besten Kenner der Leberatrophie, UмBะR ${ }^{2}$ ), 3 Fälle von Leberatrophie zur Operation gebracht hat, weil für Choledochussteinverschlu $8 \mathrm{bzw}$. schwere Cholangitis sprechende Symptome bei sicher diagnostizierter Leberatrophie vorhanden waren.

Abgesehen von den Umberschen Fällen, die NEUPERT operierte, sind noch einige weitere Fälle von Leberatrophie in den letzten Jahren laparotomiert und durch die Operation sichergestellt worden, und zwar von $\mathrm{K}_{\text {A USCH, }} \mathrm{Z}_{\text {ELLER, ALKAN, }}$

*) Vortrag, gehalten in der Berliner Chirurg. Gesellschaft 16, Oktober 1922 .
TiEtze. Ich selbst kann 5 Fälle meiner Abteilung hinzufügen, in denen wir uns bei schwerer Parenchymerkrankung der Leber zur Operation gezwungen sahen. Ich gebe nur kurze Daten über die Kranken: Meine beiden ersten Fälle zeigten die Kombination von chronischer Cholangitis calculosa mit akuter Leberatrophie bzw. Lebernekrose bei Frauen mittleren Alters.

I. In der Vorgeschichte von Fall I fanden sich ro Gallensteinanfälle. Die Erkrankung dauerte 4 Wochen, der Ikterus I4 Tage. Die Operation beschränkte sich auf die Entfernung der steinhaltigen Gallenblase. Der Leberbefund war uncharakteristisch; ihre Farbe rotbraun mit ikterischem Grundton, ihre Konsistenz brüchig, Größe und Oberfläche anscheinend normal. Die Gänge frei von Steinen, zartwandig, nicht erweitert. Aus dem Cysticusstumpf entleerte sich nur wenig gelblicher Schleim. Da weder eine Stauung noch Entzündung des Hauptgallenganges vorlag, und der Ikterus auf eine primäre Parenchymerkrankung der Leber bezogen wurde, Verzicht auf Hepaticusdrainage. Tod bereits am nächsten Tage. Autopsie ergab schwere gelbe Leberatrophie, schwerste Parenchymnekrose, reichliche Gallengangswucherungen. Die Leberkonsistenz war äußerst schlaff und weich, besonders in einem mannsfaustgroßen Bezirk des r. Lappens (L. PICr).

2. Im zweiten, bereits 1905 beobachteten Falle mit 7 wöchiger Krankheitsdauer wurden zwei größere Steine aus Gallenblase und Cysticus entfernt, Gallenblase und zartwandige. nicht erweiterte Choledochus wurden drainiert. Die Farbe der Leber bläulich, r. Leberlappen bis unter den Rippenbogen hinabreichend, in seinem unteren Abschnitt grauweise, strangförmige Kapselverdickungen, so daß die Oberfläche etwas gekörnt und uneben erschien. Aus dem Choledochus floß nur wenig zähe Galle; der Gallenfluß kam nicht mehr in Gang. Nach 5 Tagen Tod unter cholämischen Erscheinungen. Die Autopsie zeigte KIeinheit der Leber, normale Konsistenz und intensive ikterische Färbung. Mikroskopisch (K. HART) zentrale Nekrose der Leberläppchen.

Fall 3 und 4 kamen wegen Verdacht auf aufsteigende Cholangitis zur Operation.

3. Davon betraf die eine $2^{1 /}$ Jahre zurückliegende Beobachtung ein 33 jähriges Mädchen. 12 Wochen vor der Operation Erkrankung unter schweren Magen- und Darmerscheinungen, 3 .Wochen später Ikterus. Der scharfwandige Leberrand reichte zuerst bis zum Nabel. Es bestanden Schmerzen in der Lebergegend, Fieber- und Schüttelfröste traten auf. Der Stuhl war entfärbt. Nach zunächst abwartender Beobachtung auf der Zweiten Internen Abteilung (MAGNUS-LEVY) und der Chirurgischen Abteilung Operation wegen Verschlechterung des Befindens und erneuter Schüttelfröste mit Fieber bis $39^{8}$. Exstirpation der wenig veränderten Gallenblase, Drainage des zartwandigen, nicht erweiterten Choledochus, aus dem sich nur spärliche, dünne, gelbe Galle entleerte. Die Leber war nicht mehr vergrößert, ihre Oberfläche glatt, Farbe blaß-braun. Die mikroskopische ${ }^{3}$ Untersuchung eines Leberstückchens ergab das Bild der akuten Leberatrophie (L. PICK). 
Die Menge der abgeleiteten Galle stieg bereits am nächsten Tage auf 650, im späteren Verlauf bis auf $1700 \mathrm{ccm}$ täglich. Langsame $\mathrm{Er}-$ holung. Etwa 2 Jahre nach der Operation keine Leberstörungen.

4. Im 4. Falle, 39 jähriger Mann, Krankheitsanfang vor 15 , Ikterus seit 4 Tagen bei mäßigem Fieber. - Wegen des schlechten Zustandes Operation durch WorTManN bereits am nächsten Tage. Exstirpation der prall gefüllten, sonst nicht veränderten Gallenblase. Drajnage des nicht erweiterten, steinfreien Choledochus, aus dem sich völlig farblose Galle in spärlicher Menge entleerte. Leber wenig vergröBert; Oberfläche etwas hyperämisch mit heller gefärbten gelblichen Inselchen. Untersuchung eines Leberstückchens (L. PICK) ergab Zerfall und Defekt von Leberzellen mit kleinzelliger Infiltration an einzelnen Stellen, keine deutliche Gallengangswucherung. Auch hier trat bald ein lebhafter Gallenfluß ein. Die Gaile war bereits am Tage nach der Operation intensiv gefärbt. Auch hier 4 Monate nach der Operation guter Zustand, keine Leberstörungen.

5. Der Vollständigkeit halber sei noch eine Beobachtung ( 8 .jähriges Mädchen) von tödlicher Blutung aus einem Corpus luteum bei schwerer Leberatrophie erwähnt. Die Operation beschränkte sich auf Blutstillung im Unterbauch.

Den operativen Befund an der Leber habe ich ausführlicher gebracht, um zu zeigen, daß er in meinen Fällen nicht genügte, die Diagnose einer schweren Parenchymerkrankung der Leber wesentlich zu stützen. Anders war das in Fällen, die NEuperT und $\mathrm{KAUSCH}$ operierten.

In NeUPERTs einem Fall zeigte sich zwei Monate nach Beginn der Erkrankung bei der Operation die Leber im Zustande starker Degeneration; sie war verkleinert, blaßrot gefärbte knotige Vorwölbungen von $1 / 2-3 / 4 \mathrm{~cm}$ Höhe und zum Teil cavernomähnlichem Aussehen lagen zwischen rötlichen atrophischen Leberpartien. In $\mathrm{K}_{\mathrm{AUSCH}} \mathrm{S}^{4}$ ) Fall mit Ascites war $\mathrm{I}^{1 / 2}$ Monate nach Krankheitsbeginn die Leber klein, ihre Farbe dunkelbraun, die Oberfläche gleichmäßig dicht granuliert, so daß ein cirrhotischer Prozeß angenommen wurde. KAUSCH drainierte in diessm Falle ausgiebig den Ascites, NEUPERT beschränkte sich auf die Probeexcision. Beide Fälle genasen wie meine Fälle 3 und 4 .

Im Gegensatz zu dem uncharakteristischen Leberbefund wies in meinen Fällen der negative Befund am Hauptgallengang, seine Zartheit und die fehlende Erweiterung ebenso wie die Spärlichkeit und veränderte Beschaffenheit der Galle auf eine degenerative Parenchymerkrankung der Leber hin.

Bei der Bewertung der Heilwirkung operativer Eingriffe bei Leberatrophie $\mathrm{muB}$ man sehr vorsichtig sein. Abgesehen von den von NeUPerT und KaUSCH nach einfacher Probelaparotomie bzw. Ablassen des Ascites beobachteten Genesungen mehren sich in den letzten Jahren die Fälle von Genesung nach subakuter Leberatrophie bei rein interner Behandlung (UMBER, STRÜMPELL usw.). Ich selbst sah ebenfalls einen hierher gehörigen Kranken ohne Operation zur Heilung kommen.

Daß wir in unseren 4 Fällen durch die Laparotomie eine Klärung herbeiführen mußten, halte ich für zweifellos. Strittig kann nur sein, ob wir uns bei dem negativen Befund am Hauptgallengang auf die Probelaparotomie und Probeexcision zur Sicherung der Diagnose hätten beschränken sollen. Ich glaube, das wäre nicht richtig gewesen. Einmal mußten wir mit der Möglichkeit rechnen, daß die Leberschädigung, zum Teil wenigstens, auf das tatsächlich vorhandene Steinleiden bzw. auf eine Entzündung der kleineren Gallengänge zurückzuführen war. Auf das häufige Vorkommen von Cholangie der kleinen und kleinsten Gallengänge bei Leberatrophie haben UMBER und Versè besonders hingewiesen.

Ich halte aber zweitens eine direkte Heilwirkung der Hepaticusdrainage auf die Parenchymerkrankung der Leber für durchaus möglich. Die schnelle Zunahme der abgeleiteten Galle in dem einen, die Zunahme und rasche Wiederkehr normaler Färbung der Galle in dem anderen meiner geheilten Fälle und die Wendung zum Besseren nach der Operation nach vorher sehr bedrohlichem Zustande sprechen dafür. Im gleichen Sinne hat sich kürzlich TIETZE ${ }^{5}$ ) für Fälle von Leberatrophie, die sich im Anschluß an eine Infektion entwickeln, ausgesprochen. TIETzE sah in zwei von drei hepaticusdrainierten Fällen Besserung. Nach TIETZE wird die $\mathrm{Ab}$ schwellung der Leber durch die Drainage begünstigt. Tintze denkt außerdem daran, daß der durch Einlegen eines Rohres in den Gang ausgeübte funktionelle Reiz die Regenerationsvorgänge in der. Leber günstig beeinflußt. Ich stelle mir die Wirkung so vor:

I. Wir können - besonders in Fällen mit Cholangie der kleinen und kleinsten Gänge - durch die gleichmäßige dauernde Absaugung der vorher unter minimalem Druck stehenden, stagnierenden Galle eine wesentliche Entlastung und Erholung des Leberparenchyms herbeiführen.

2. Mit der reichlicher fließenden Galle wird gleichzeitig wenigstens ein Teil der hochgiftigen autolytischen Zerfallsprodukte der Leberzellen mit fortgeführt, ihre Resorption verhindert und die Vergiftungsgefahr vermindert.

3. Können wir bei vollständiger Ableitung der Galle nach außen die Resorption der Gallenbestandteile aus dem Darm, also den sog. Gallenkreislauf, für einige Zeit vollständig ausschalten und auch hierdurch wieder zur funktionellen Entlastung des insuffizienten Leberparenchyms beitragen.

4. $\mathrm{Ob}$ wir außerdem noch mit nicht näher bekannten reflektorischen Einflüssen zu rechnen haben, muß ich dahingestellt sein lassen.

Diese Erwägungen führen mich zu dem Schluß, daß man in fortschreitend ungünstig verlaufenden Fällen von Leberatrophie und nekrotisierenden Parenchymerkrankungen der Leber, auch wenn ein oder eine Steinleiden aufsteigende Cholangitis differentialdiagnostisch nicht in Betracht kommen, die Hepaticusdrainage versuchen soll. Die Indikation muß in enger Fühlung mit den Internisten, aber nicht zu spät, d. H. nicht erst, wenn das ganze Parenchym bereits zerstört ist, geschehen. Bei den schweren akut verlaufenden Formen der Leberatrophie dürfen wir wenig hoffen; aber auch hier dürfte ein Versuch berechtigt sein. Ein abschließenden Urteil ist erst bei Vorliegen gröBerer Erfahrungen möglich.

Folgendes muß noch besonders unterstrichen werden: Alle Eingriffe an den äußerst labilen Kranken mit Leberatrophie mußten mit größter Behutsamkeit und Schnelligkeit ausgeführt werden. Kein Chloroform! Kein unsicheres zeitraubendes Absuchen der zarten Gänge! Wegen der Gefahr der cholämischen Blutung und zur Abkürzung des Eingriffs möglichst Verzicht auf Gallenblasenexstirpation und Tamponade des Wundbettes! Schneller SchluB der Bauchwunde durch einige durchgreifende Nähte. Durch reichliche subcutane oder intravenöse Kochsalzinfusionen muß schließlich die Leber und der ganze Körper durchspült und ausgewaschen und damit gleichzeitig die Gallenmenge gesteigert werden.

Anhangsweise teile ich noch eine Beobachtung mit, die mir H. Schum aus dem Staatskrankenhaus der Polizei, Berlin, zur Verfügung stellte.

22 jähriger Mann. Vor I92 I kurz nach Beginn einer Salvarsankur leichte kolikartige Schmerzen in der Lebergegend. Fünfmalige Wiederholung. Im Februar I922 heftigere Wiederholung der Anfälle. März rg22 Ikterus. Vor der Operation starker Ikterus, heftige Koliken, acholischer Stuhl. Operation ergab derbe, etwas geschwollene Leber. Gallenblase frei von Konkrementen, enthält nur etwas helle, zähe Flüssigkeit. Gallenwege zartwandig, nicht erweitert; Sondierung nach oben und unten leicht möglich. Nirgends ein Stein oder sonstiges Hindernis. In den Wegen nur ganz wenig helle, dicke Flüssigkeit. Exstirpation der Gallenblase, Hepaticusdrainage. Schon am Tage nach der Operation wesentlich hellere Farbe des Urins; am 4. Tage leicht gallige Färbung des Stuhls, dann sehr starker Abfluß erst hellerer, dann dunkler Galle aus dem Drain; Urin nach I2 Tagen völlig frei \&von Gallenfarbstoff. Stuhl bald normal gefärbt. Heilung bis auf geringe Beschwerden in der Lebergegend.

Literatur: 1) HerXhermer, Uber die akute gelbe Leberatrophie. Klin. Wochenschr, I922, S, I44I. - 2) UMBER, Zur Klinik der akuten bzw. subakuten Leberatrophie. Dtsch. med. Wochenschr. I9I9, S. 537. $-^{3}$ ) UMBER, Akute und subakute Leberatrophie. Klin. Wochenschr. 1922, S. I $585 .-4)$ HubER und KaUsCH, Zur Klinik der subakuten Leberatrophie. Berl. klin. Wochenschr. I920, S. 8 I. - ${ }^{5}$ ) Tierze, Zentralbl. f. Chirurg. I922. Nr. 27. 